\title{
Health Promotion among Mentally Ill People in Nepal
}

\author{
Ghimire $S^{1}{ }^{1}$, Dhungana $M^{2}$, Bhagat $S^{3}$, Parajuli $S^{4}$
}

1. Lecturer in Department of Psychiatry, National Medical College, Birgunj, 2. Consultant Psychiatrist, Crimson Hospital, Rupandehi, 3. Clinical observer, St. Mary's Hospital, Decatur, IL, USA, 4. Kathmandu National Medical College, Anamnagar, Kathmandu(Proposed)

E-mail *Corresponding author: drsrghimire@gmail.com

\begin{abstract}
Mental health is a neglected area and the activities to promote mental health are limited in Nepal. Exploration of various social determinants of mental health is needed prior to starting mental health promotion that do not only lie within health sector but also deeply rooted outside. Mental health promotional and preventive program needs to be locally relevant, culturally appropriate and cost effective, and require social and public health approaches. Provision of community mental health strategy to the door step of people can be an effective strategy to promote health among mentally ill population. Health sector needs to be coordinated and collaborated with other sectors to address the complex mental health problems. Consideration of cultural and religious aspect in health care systems is essential to ensure that every community are equally respected and are treated equally despite variation.
\end{abstract}

Key words: Health Promotion, Mentally ill, Nepal

\section{INTRODUCTION}

Mental health is defined by WHO as "a state of well-being in which the individual realizes his or her own abilities, can cope with the normal stresses of life, can work productively and fruitfully, and is able to make a contribution to his or her community". ${ }^{1}$ Mental health and well being is neglected in and given less emphasis in the context of Nepal. ${ }^{2}$ This article tends to highlight the different social determinant of mental health such as poverty, war, social, religious and cultural factors in the context of Nepal which need more priority and attention. Then it will highlight the need for implementation of health promotion strategy that will help to address this issue. The important strategy to address these issues in this context is determined as strengthening health care systems by promoting health in all sectors and settings and assuring universal access to mental health care.

\section{Social Determinants and Mental Health}

\section{War and mental illness}

The impact of the war in mental health has been significant. Many are victims of war and are recovering from a decade long political conflict which took the lives of thousands of people and left many affected. ${ }^{3}$ The residual impact of this war is slowly being felt in almost all sectors including the tourism, health, and industry. ${ }^{4}$ A gradual increase in the incidence of depression, posttraumatic stress disorder, and suicide has been noted since the beginning of the conflict. A survey done in two towns of western Nepal in 1998 revealed $35 \%$ prevalence of psychiatric morbidity. ${ }^{4}$ Similarly, another study done by Shakya et al found that conflict had affected more than half directly either psychologically and physically and the former caused the major impact in quality of life. ${ }^{5}$ The consequences of the war have made the issue of mental health and illness more complex by 
adding on the previously persistent cultural and social beliefs revolving around the issue of mental illness.

Depending on existent protective and risk factors, living in situations of political violence can result in (a) compromised psychosocial wellbeing/mental health (i.e. suffering which does not lead to impairment in daily activities), (b) psychological distress (i.e. emotional anguish accompanied by impairment in daily activities), or (c) mental disorders (i.e. specific psychiatric suffering meeting diagnostic criteria). ${ }^{4}$ In a study done among internally displaced persons during armed conflict in Nepal, it was found that $80.7 \%$ males suffered from anxiety and $80.3 \%$ from depression $(90.3 \%$ and $88.5 \%$, respectively, in females). ${ }^{6} \mathrm{~A}$ study showed that the more violent or inhuman the experience, the worse the impact on mental health. ${ }^{7}$ Integration of mental health services into primary health care can only be the cost effective, large scale measure to provide care and support to such people. ${ }^{8}$

\section{Poverty and mental health}

The relationship between poverty and mental disorders has a vicious cycle ${ }^{9}$ and one leads to the other. Depressive and anxiety disorders are consistently shown to be present in poor and marginalized people who are at greater risk of suffering from these. ${ }^{10}$ Poverty and socioeconomic problems have been cited as one of the most important factors causing emotional distress. The association between poverty and common mental disorders is considered as a universal one, occurring in all societies irrespective of their levels of development. ${ }^{11}$ The psychological impact of living in poverty is mediated by shame, stigma and the humiliation of poverty..$^{9}$ Factors such as the experience of insecurity and hopelessness, rapid social change and the risks of violence and physical ill-health may explain the greater vulnerability of the poor to common mental disorders. ${ }^{11}$ It is less likely that a direct causal effect of traumatic events creates symptomatology, rather mental health and wellbeing is associated with sociocontextual factors such as poverty, gender, education and social connectedness. ${ }^{12}$
A study done in the western region of Nepal explored the high prevalence of psychiatric illness in the rural community..$^{13}$ As majority of rural population are under poverty level, the study pointed out unemployment, unskilled job among other caste (Dalit/Nepali) and the use of alcohol among them contribute to mental illness. ${ }^{13}$ Females are vulnerable for developing mental illness due to unemployment, dependency on males, and also due to male dominant societal system which forced them to involve in sex profession to feed themselves and their family. A study of the mental health of Nepalese women found that the commercial sex workers group showed a higher prevalence of anxiety and depression than the non-sex workers group and majority of them presented with somatic symptoms. ${ }^{14}$ They had been found to be having more difficult social situations than the non-sex workers group. Thus the reasons for their being sex work is poverty. ${ }^{15}$

\section{Social, religious beliefs and mental health}

Mental well-being for many people in Nepal is linked to their relationship with their concept of God.16 Problems are considered to be caused by a breakdown in this relationship or an oversight in the respect that should have been shown..$^{10}$ Consequently, problem-solving requires some action that repairs this relationship. Mentally ill people are taken to natural healers rather than to modern health care facilities. ${ }^{17}$ On the other hand, it is not uncommon to visit traditional healers and general physicians simultaneously. The phenomenon of hierarchy of resort dependent on a range of issues in addition to traditional religious beliefs, including class, caste and ethnicity, educational standing, and economic status. ${ }^{18}$ Apart from this the most basic cultural and moral barrier to the amelioration of mental health problems continues to be the enormously negative, destructive, and almost universal stigma that is attached to mental illnesses, to patients with a mental illness and their families. ${ }^{18}$

STRENGTHENING HEALTH CARE
SYSTEMS TO PROMOTE HEALTH
AMONG MENTALLY ILL PEOPLE




\section{Provision of mental health services}

Mental health services, which were rudimentary to begin with, have been further fragmented by the recent civil war. At present there are only 37 community-based psychiatric inpatient units available. ${ }^{19}$ The role of the government to increase community mental health awareness is by providing adequate services at all regions of the country. This can be done by integrating mental health services into primary health care. Integrating mental health services into primary health care helps to provide effective mental health care in countries which have inadequate resources to address the burden of mental disorders properly. ${ }^{17}$ In addition, this provides opportunities for people who generally prefer to contact primary care centers rather than institutes dealing with mental illness alone because of the social stigma attached to it. ${ }^{20,21}$ This will thus result in detection, treatment and support to mentally ill patients and their families. However, on the other hand there are difficulties and impracticalities of integration of mental health services into primary health care in low resourced countries. ${ }^{3}$

The three main concerns that Patel ${ }^{12}$ points out while including mental health services in primary health care are a)Inadequate training of the primary health care workers in detecting the psychological symptoms in patients who present with somatic symptoms and because they do not have enough time to elicit psychological symptoms. To maximise case detection, the government provides training to the service providers and arranges for referral of severe cases to specialized tertiary care centres. ${ }^{21}$ b) Improper use of psychotropic drugs, for instance the inadequate use of evidence-based medications and the frequent use of non-evidence-based medications. The government of Nepal has placed effort on providing specific assessment and treatment protocols for key mental health conditions. These protocols are being implemented in about $21-50 \%$ ) physicianbased primary health care clinics, and around $1-20 \%$ of non-physician based primary health care. Primary health care workers other than doctors such as -nurses, non-doctor/nonnurse primary health care workers are allowed to prescribe but with restrictions. ${ }^{21}$ c) Stigma associated with mental disorders and the lack of availability of psychosocial interventions to address the social determinants of mental disorders. As the literature suggests, stigma associated with mental illness is very strong. There has not been adequate effort from the government to bring awareness about stigma related to mental illness. ${ }^{20}$ The government's role is to implement the national health policy and provide clear guidelines so that mentally ill people are included in the society. ${ }^{22}$ There is a need for collaboration across a range of services provided or funded by different government and private sectors, nongovernment agencies, individuals and organisations in the community to improve the mental health. ${ }^{23}$ There is some evidence to suggest that integration of mental health care into routine primary care has been and can be sustainable and effective. ${ }^{8}$

Community mental health services are limited to a few places in Nepal. The development of a national community mental health program is most important issue for the Ministry of Health to address. ${ }^{24}$ Community care facilitates the empowerment of people with mental and behavioural disorders. It implies the availability of a wide range of culturally acceptable and cost effective services within local settings. These community based care centers ensure that the protective functions are fully provided in the community while the negative aspects of the institutionalisation are discouraged.25 Community mental health services can be strengthened with the partnership of national government and NGOs. These health services have to be made available at the regional, district and peripheral levels.

WHO describes community based care as an approach, which provides services close to home, including general hospital care for acute admissions, and long-term residential facilities in the community; treatment and care specific to the diagnosis and needs of each individual; and partnership with carers and meeting their needs; legislation to support the above aspects of care. ${ }^{1}$ A balance of community-based and hospital-based services has been shown to be the most effective form of comprehensive mental health care. ${ }^{26}$ Evidence suggests that, in low-income and middle income countries, support for primary care services to enable them to identify and treat people with mental disorders, with 
training, assistance, and supervision by available specialist mental health staff, is the best way to extend mental health care to the population. ${ }^{26}$ Study on health service evaluations have shown that communitybased care models, such as community-based rehabilitation and community outreach programs in India, are able to reach out to people in rural and impoverished communities, producing tangible benefits in terms of improved clinical outcomes, reduced levels of disability and reduced family caregiving burden. ${ }^{27}$ This approach may be applied in the context of Nepal.

\section{Provision of cultural competency, social and religious aspect in health care systems}

The World Health Organisation places the value of religious and spiritual aspect of mental health. WHO emphasized the need to understand and respect the spiritual aspect of mental health as defined by both individuals and cultures. ${ }^{28}$ Health care systems should assure universal access to health services including mentally ill population who rely and believe on traditional beliefs and practices. ${ }^{25}$ Due to strong belief in traditional healing, the first contact of many mentally ill patients is traditional healer rather than health professionals. Thus involvement of these people in mental health activities is essential to achieve the mental health goal. Since, there is lack of human resources in mental health, available resource is inadequate to bring about rapid awareness about mental health and illnesses. In this context, one probable alternative can be the utilization of traditional healers after providing them mental health promotion training.

Health care providers must be trained to understand traditional beliefs and practices to acknowledge and respect peoples' beliefs and practices in order to increase the use of health care among them. ${ }^{28}$ Health promotion and service delivery should consider cultural beliefs and practices and need to involve communities in planning, implementation and evaluation of health promotion and services may be an effective strategy to meet the need of mentally ill people.25 Religious leaders, socially famous person should be involved in health care delivery system for optimal outcome. ${ }^{10}$ Furthermore, cultural competency education should be included in school and college curriculum to make understand the mental health and to decrease stigma related to it.

\section{Multisectoral collaboration}

Mental health problem is rooted everywhere in Nepalese society and the determinants of this issue is vague. There is need of multisectoral collaboration and co-ordination to solve this problem. ${ }^{30}$ Most of the factors lie outside the health sector and the need of addressing it is crucial. WHO recommends need of collaboration between different sectors within the country and also pointed out the need of it between the mental health resourced and poorly resourced countries to share experience and provide international expertise experience. ${ }^{28}$ Strong political commitment to mental health care and in the multilateral partnerships marshaling the resources to improve mental health in countries with limited resources. ${ }^{18}$ For instance there is need of collaboration with housing department to solve the problem of homelessness, poor and displaced population and victim of conflict. Collaboration with different agencies along with integrating mental health services into primary health care is the better option which helps to provide effective mental health care in countries which have inadequate resources to address the burden of mental disorders properly. ${ }^{18,31}$ Similarly collabarative models of "task shifting" in the delivery of health care, for instance, using community health workers in low-income settings for health promotion activities have been found successful. ${ }^{32}$ Several initiatives are needed including funding, and attention towards addressing the mental health need which is possible only with collaborative partnership with government, local NGOs and international agencies. ${ }^{18}$ Provision of income generation activities among women and men help to minimize poverty ${ }^{31}$ and that lead to decrease incidence of mental problem. ${ }^{32}$ Similarly alternate income generation activities among sex workers will encourage to them to quit that profession and minimize the mental health problems. ${ }^{15}$ 


\section{CONCLUSION}

Various social determinants of mental health play a key role and these do not only lie within health sector but also rooted outside the health sector. Strengthening health care systems by provision of community mental health strategy can be an effective strategy to promote health among mentally ill population. Health care systems should embed health in all policies to ensure that all sectors' decision makers aware of the impacts of their policies on mental health. Health sector needs to be coordinated and collaborated with other sectors to address the complex mental health problems. Multidimensional approach is crucial to overcome this issue. Finally, considering cultural and religious aspect in health care systems is essential to ensure that every community are equally respected and are treated equally despite of variation. Involving different community in planning, implementation and evaluation of health promotion strategies and programs is critical to promote health among mentally ill population.

\section{REFERENCE}

1. World Health Organization. Mental Health, Human Rights \& Legislation : WHO's Framework. 2001[Cited 2014 June10]. Available from: www.who.int/mental_health/policy/fact_sheet_m nh_hr_Leg_2105.pdf

2. Devkota M. Mental health in Nepal: the voices of Koshish. American Psychological Association. Washington DC.2011[Cited 2014 sept 10]. Available from:

http://www.apa.org/international/pi/2011/07/ne pal.aspx

3. Tol WA, Kohrt BA, Jordan MJ, Thapa SB. Political Violence and Mental Health : A Multi- Disciplinary Review of The Literature of Nepal. Social Science Medicine. 2010;70(1):35-44.

4. Upadhayaya K, Pol N. Survey of Psychiatric Morbidity in Arghakhanchi and Gulmi districts of western of Nepal ,Collaboration with NPA.2003

5. Shakya DR, Lamichhane N, Shyangwa PM, Shakya R. Nepalese psychiatric patients with armed-conflict related stressors. Health Renaissance.2011;9(2):67-72.

6. Thapa SB and Hauff E. Psychological distress among displaced person during an armed conflict in Nepal, Social Psychiatry. 2005; 40:672-91.

7. Acharya L, Upadhyay KD, Kortmann F. Mental Health \& Psychosocial Support Aspects in Disaster Preparedness in Nepal. Int Rev Psychiatry. 2006;18(6):587-92.
8. Cohen A. The effectiveness of mental health services in primary health care. The lancet. 2001 9. Patel V, Kleinman KA. Poverty and common mental disorders in developing countries, International Journal of Public Health. 2003;81(8):609-15.

10. Devkota B. Culture \& Mental Health. Journal of Nepal Medical Association.2005;34(122): 67-73.

11. Patel V, Saraceno, Kleinman A. Beyond evidence: The moral case for international mental health. AMJ Psychiatry.2003;163(8):1312-15.

12. Patel V. Mental health in low and middle income countries. British Medical Journal.2007

13. Khattri JB, Poudel BM, Thapa P, Godar ST, Tirkey S, Ramesh K, Chakrabortty PK. An Epidemiological Study of Psychiatric Cases in a Rural Community of Nepal. Nepal Journal of Medical Sciences 2013;2(1):52-6.

14. Eller LS, Mahat G. Psychological factors in Nepali former commercial sex workers with HIV. J Nurs Scholarsh. 2003;35(1):53-60.

15. Seale S. Challenges faced by women living with $\mathrm{HIV} / \mathrm{AIDS}$ in India, Indian female right Journal.2009;5:12-25.

16. Kohrt BA, Harper I. Navigating Diagnoses ,understanding Mind- Body Relations, Mental Health \& Stigma in Nepal, Cult Med Psychiatry. 2008;32(4):462-91.

17. Jha, A, Adhikari SR. Mental Health Services in Nepal-Observations, Objections \& Outlooks for the future. Journal of Nepal Medical Association. 2009;48(174):185-90.

18. Becker AE, Kleinman A. Mental Health and the Global Agenda. N Engl J Med. 2013; 369:66-73.

19. Regmi S, Pokharel A. Nepal Mental Health Profile,TUTH, Kathmandu,Nepal. 2010

20. Regmi SK, Pokhrel A, Ojha SP, Pradhan SN, Chapagain G. Nepal mental health country profile. International Review of Psychiatry. 2004;16(12):14249.

21. World Health Organization. WHO-AIMS Report on Mental Health System in Nepal. 2006 [Cited 2014 June 2].Available from:

www.who.int/mental_health/evidence/nepal_wh o_aims_report.pdf

22. Segall M. District Health Systems in a neoliberal World: a review of five key policy areas. International Journal of Health Planning \& Management. 2003;18:S5-S26.

23. Green A. The policy context: an introduction to health planning for developing health systems. Oxford University Press.2007;3:56-69.

24. Shyangwa PM, Jha A. Nepal: trying to reach out to the community. International Psychiatry. 2008; 5(2):36-38.

25. Thara R., Ramesh kumar S., CG. M. Publications on community psychiatry. Indian J Psychiatry. 2010;52 (Suppl 1):274-7.

26. Saxena, S. and Thornicroft G. Resources for mental health: Scarcity, inequity and inefficiency. Lancet. 2007:878-89. 
27. Chatterjeee S, Pillai A, Jain S, Cohen A, Patel V. Evaluation of community based rehabilitation model for mental disorder in rural India, The British Journal of Psychiatry.2003; 195(5):433-39.

28. World Health Organization. Promoting Mental Health. World Health Organization. Geneva. [Cited 2014 oct 21] Available from: http://www.who.int/mental_health/evidence/en /promoting_mhh.pdf.

29. Baum F. The new public health. Oxford University Press, Melbourne.3rd edition. 2008

30. Subedi J. Modern health services and health care behavior :A survey in Kathmandu, Nepal. Journal of Health and Social Behavior.1999;30:412-20.

31 Patel, V. The future of psychiatry in low- and middle-income countries. Psychol Med. 2009;39:1759-62.

32. Kabeer N. Conflicts over credit: re-evaluating the empowerment potential of loans to women in rural Bangladesh. Conflict over credit. 2001; 29(1):63-84. 